\title{
PROARRHYTHMIC EFFECTS OF ANTIARRHYTHMIC DRUGS ASSESSED BY ELECTROPHYSIOLOGIC STUDY IN RECURRENT SUSTAINED VENTRICULAR TACHYCARDIA
}

\author{
Masaomi Chinushi, M.D., Yosifusa Aizawa, M.D., Seitchi Miyajima, M.D. \\ Toshikazu Funazaki, M.D., Makoto Tamura, M.D. \\ and Akira Shibata, M.D.
}

\begin{abstract}
Proarrhythmic responses were evaluated in repeated electrophsiologic studies (EPS) in 27 patients with inducible ventricular tachycardia (VT). Class Ia drugs were administered to 23 , Ib to 6 , Ic to 4 , III to 5 and IV to 9 patients. The mean age was 53 years, and 18 patients had structural heart diseases. Pleomorphism was observed in 11 patients. In 4 patients $(15 \%)$, the VT cycle length (CL) shortened by $50 \mathrm{~ms}$ or more in EPS during the administration of antiarrhythmic drugs. VT was inducible by a less aggressive induction mode than the control study in 9 patients $(33 \%)$ ). In 4 patients $(15 \%)$, the induced VT changed to the incessant form, and the other 2 patients $(7 \%)$ required DC shocks due to hemodynamic deterioration. Patients with pleomorphic VT and/or structural heart diseases seemed to develop proarrhythmia more frequently. In total, some proarrhythmic response was observed in $13(48 \%)$ of the 27 patients. Therefore, it should be kept in mind that proarrhythmic effects are frequently observed during antiarrhythmic therapy in patients with sustained VT. The action of the drugs on the slow conduction zone may vary, which may provide a basis for the development of proarrhythmia.
\end{abstract}

$\mathbf{I}^{\mathrm{N}}$ addition to pharmacological therapy ${ }^{1-3}$ and surgical ablation, catheter ablation $^{5,6}$ and the automatic implantable cardioverter-defibrillator $^{7-9}$ have also been recently applied to the therapy of sustained ventricular tachycardia (VT). The first step for the treatment of VT is usually pharmacological therapy, but antiarrhythmic drugs have both suppressive and aggravating proarrhythmic effects. ${ }^{10-13}$ In this study, the incidence of proarrhythmia and the clinical characteristics of patients who developed proarrhythmia during repeated electrophy-

Key words:

Sustained ventricular tachycardia

Antiarrhythmic drug

Electrophysiologic study

Proarrhythmia siologic studies were evaluated in patients who experienced sustained VT.

\section{SUBJECTS AND METHODS}

\section{Study patients}

Sixty patients who experienced sustained ventricular tachycardia (VT) underwent electrophysiologic studies (EPS) at Niigata University Hospital from 1982 through 1988. In 27 patients ( 24 men, 3 women), VT was induced both in the control and in the drug tests. Proarrhythmic effects were evaluated in these 27 patients. Eighteen patients had structural heart diseases of which 6 had previous myocardial infarction, 7 arrhythmogenic right ventricular dysplasia, 2 idiopathic dilated cardiomyopathy, 2 hyper-

(Received November 13, 1989; accepted April 16, 1990)

The First Department of Internal Medicine, Niigata University School of Medicine, Niigata, Japan

Mailing address: Masaomi Chinushi, M.D., The First Department of Internal Medicine, Niigata University School of Medicine, Asahimachi, Niigata 951, Japan 
trophic cardiomyopathy, and 1 alcoholic cardiomyopathy. Sixteen patients had single monomorphic VT and 11 had pleomorphic VTs which were documented using surface 12-lead electrocardiograms (ECG). Cardiac catheterization including coronary angiography was performed in 20 patients, and the left ventricular ejection fraction was obtained from the left ventriculograms. In 7 patients, cardiac function was assessed by echocardiography. Cardiac function in the two subgroups with and without proarrhythmia was compared as described below.

\section{Electrophysiologic study (EPS)}

After obtaining informed consent, patients underwent the control EPS in a fasting and nonsedated state. All antiarrhythmic drugs were discontinued for at least 5 of their respective half-lives before the study. Multipolar electrode catheters were inserted percutaneously under local anesthesia. Positioning of the electrode catheters was done under fluoroscopic guidance.

ECG leads I, II, and V1 were simultaneously recorded with intracardiac electrograms on an ink-jet recorder (Siemens-Elema Mingograf 82) using paper speed of 100 $\mathrm{mm} / \mathrm{s}$ and stored on magnetic tape (TEAC Data recorder XR-5000).

Programmed electrical stimulation was performed by a cardiac stimulator (Fukuda Denshi CO. Cardiac Stimulater BC 02 A) with rectangular pulses of $2 \mathrm{~ms}$ duration at twice diastolic threshold. Our standard stimulation protocol included delivery of single and double ventricular extrastimuli following eight basic ventricular pacings at cycle lengths of 600 and $400 \mathrm{~ms}^{14,15}$ Incremental rapid ventricular pacing (up to 210 beats per minute) for $5-15 \mathrm{~s}$ was also performed $!^{14-16}$ In the control study, triple ventricular extrastimuli were employed in 7 patients. In the other patients, the standard protocol(single, double ventricular extrastimuli and rapid ventricular pacing) was used until VT was induced. The stimulation was performed at the right ventricular apex and at the right ventricular outflow tract. If sustained VT was not induced, isoproterenol (ISP) was infused continuously to increase the sinus rate by $20 \%$. After ISP, programmed electrical stimulations were repeated in similar manner as mentioned above. Left ven- tricular stimulation was also performed when the right ventricular stimulation failed to induce VT.

When it was possible to induce sustained VT in a control study, antiarrhythmic drug therapy was commenced. Drug selection is described below.

\section{Antiarrhythmic drug therapy}

Class Ia drugs such as procainamide $(1.5-3.0 \mathrm{~g}$ orally or $600-1000 \mathrm{mg}$ iv) and disopyramide $(300-500 \mathrm{mg}$ orally) were the drugs first chosen ${ }^{17}$ If class Ia drugs were ineffective, class Ib (mexiletine: $300 \mathrm{mg}$ orally, lidocaine: $100-150 \mathrm{mg}$ iv, aprindine: $60-80$ mg orally), class Ic (flecainide: $200-300 \mathrm{mg}$ orally), or class III (amiodarone: $200-400$ $\mathrm{mg}$ orally) were tried alone or in combination! ${ }^{18}$ In patients with idiopathic VT of RBBB and left axis deviation pattern, class IV (verapamil $120-240 \mathrm{mg}$ orally or $5-10 \mathrm{mg}$ iv) was first chosen because the VT has been known to be responsive to this drug.9-21 In total, 92 electrophsiologic studies were analysed in 27 patients in this report.

\section{Definition of arrhythmia and drug response}

1) Sustained ventricular tachycardia: VT that lasted for $30 \mathrm{~s}$ or longer, or that required termination within $30 \mathrm{~s}$ because of cardiovascular collapse. 2) Nonsustained ventricular tachycardia: VT that lasted more than 5 beats but terminated spontaneously within $30 \mathrm{~s}$. 3) Effective response to antiarrhythmic drug: successful prevention of the induction of both sustained VT and more than 15 consecutive beats using the whole protocol including the use of ISP. 4) Ineffective response to antiarrythmic drugs: failure to prevent the induction of more than 15 consecutive beats using the whole induction protocol.

\section{Definition of proarrhythmia}

Definitions for proarrythmia have not been standardized in EPS, although several criteria have been proposed? $22-24$ The following five definitions for proarrhythmia were used in this study:

(1) Induction or spontaneous recurrence of VT which has a shorter cycle length of 50 ms or more in the drug tests.

(2) Induction of sustained VT or ventricu- 
1). cycle length

2).induction mode

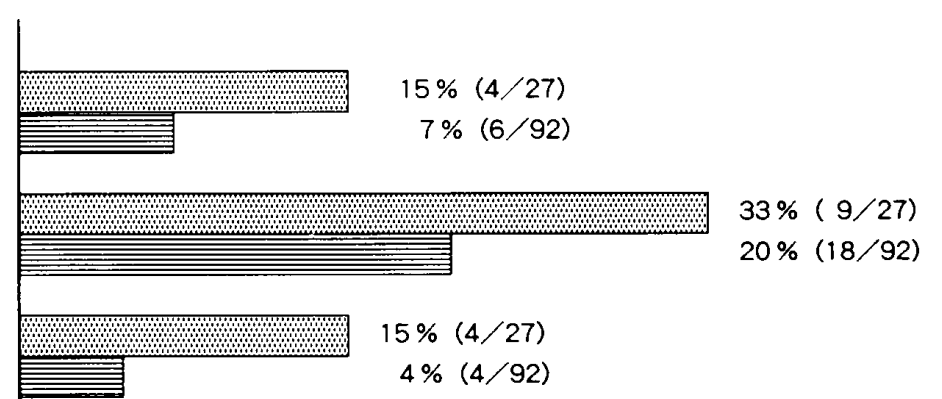

3). incessant form

4). sustained tachyarrhythmia

5). cardioversion

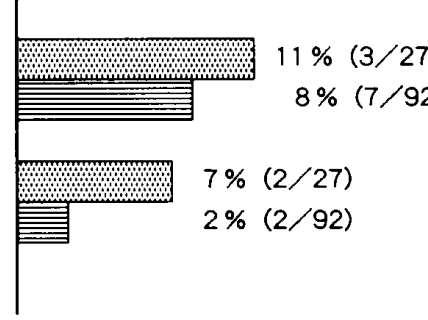

Fig.1. The incidence of proarrhythmia under each criterion. (1) Cycle length: VT cycle length shortened $50 \mathrm{~ms}$ or more in EPS using drugs. (2) Induction mode: VT became inducible with a less aggressive mode of electrical stimulation using drugs. (3) Incessant form: development of the incessant form of VT. (4) Sustained tachyarrhythmia: initiation of a sustained ventricular tachycardia or ventricular fibrillation in patients in whom only nonsustained VT was induced in the control study. (5) Cardioversion: conversion of a sustained VT that could be terminated by programmed stimulation to a sustained VT or VF which required cardioversion. For details see text.

A

control

- MuMmumum

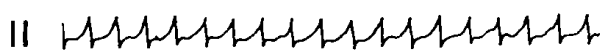

$\mathrm{CL} 286 \mathrm{~ms}$
B

I aprindine

unmminm

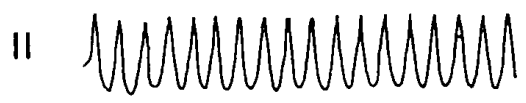

$\mathrm{CL} 230 \mathrm{~ms}$

Fig.2. A case of shortened VT cycle length. This case was a 61-year-old man with alcoholic cardiomyopathy. In A, sustained VT with a cycle length of $286 \mathrm{~ms}$ was induced by DVE $(400 / 260 / 250)$ in the control study. In B, during aprindine therapy ( $80 \mathrm{mg}$ orally per day), morphologically different VT with CL $230 \mathrm{~ms}$ was induced by DVE $(400 / 220 / 190)$. DVE=double ventricular extrastimuli. $\quad C L=$ cycle length. I, II; I, II leads of surface electrocardiogram.

lar fibrillation (VF) by less aggressive stimulation modes. These are classified as follows: conversion of the induction mode from double ventricular extrastimuli (DVE) to single ventricular extrastimulus (SVE), conversion from the rapid ventricular pacing (RP) mode to DVE or SVE, conversion from triple ventricular extrastimuli (TVE) to other modes, or lack of necessity for the use of ISP for induction after administering drugs.
(3) Development of incessant ventricular tachycardia.

(4) Conversion of nonsustained VT to sustained VT.

(5) Inability to terminate VT by the programmed stimulation after administering drugs.

\section{Statistical analysis}

Values were expressed as mean $\pm 1 \mathrm{SD}$. For statistical analysis, the t-test or chi- 
A Control

$\mathrm{CL} 220 \mathrm{~ms}$

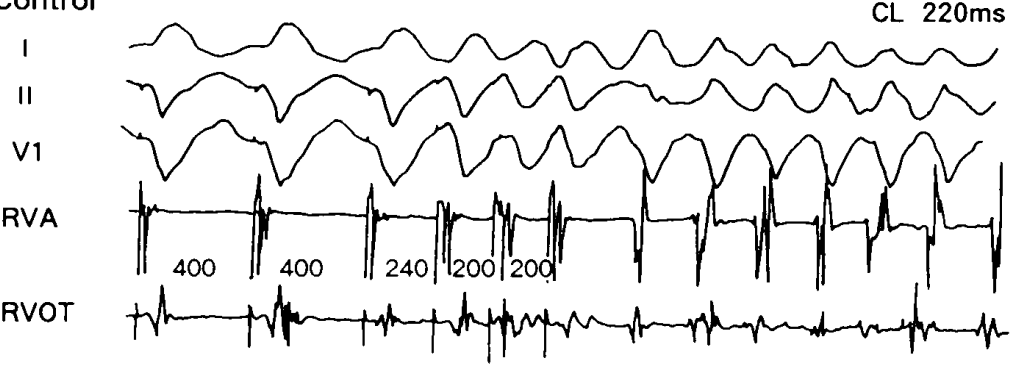

B Flecainide $200 \mathrm{mg}$

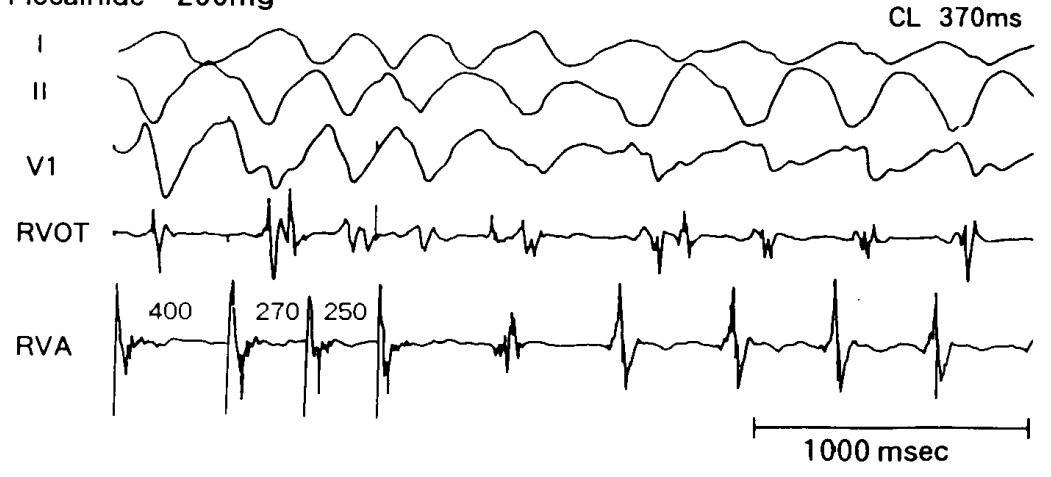

Fig.3. Change of induction mode. This case was a 55-year-old man with old myocardial infarction. VT was induced by triple extrastimuli at the right ventricular apex in the control study. During administration of flecainide ( $200 \mathrm{mg}$ orally), VT was induced by double ventricular extrastimuli at the same ventricular site. $\mathrm{RVOT}=$ right ventricular outflow tract. $\mathrm{RVA}=$ right ventricular apex. $\mathrm{S} 1=$ basic ventricular stimulation. S2, S3=ventricular extrastimuli.

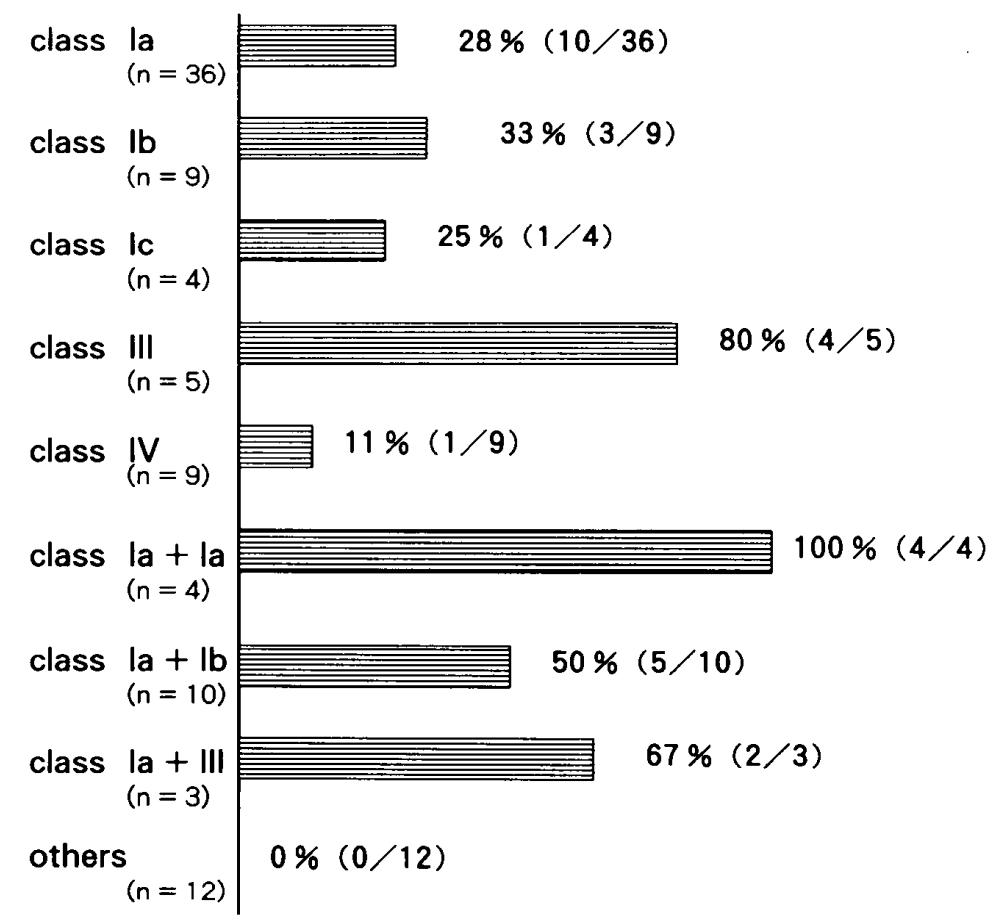

Fig.4. Incidences of proarrhythmia vs. drugs. $n=$ the number of EP trials. For details see text. 


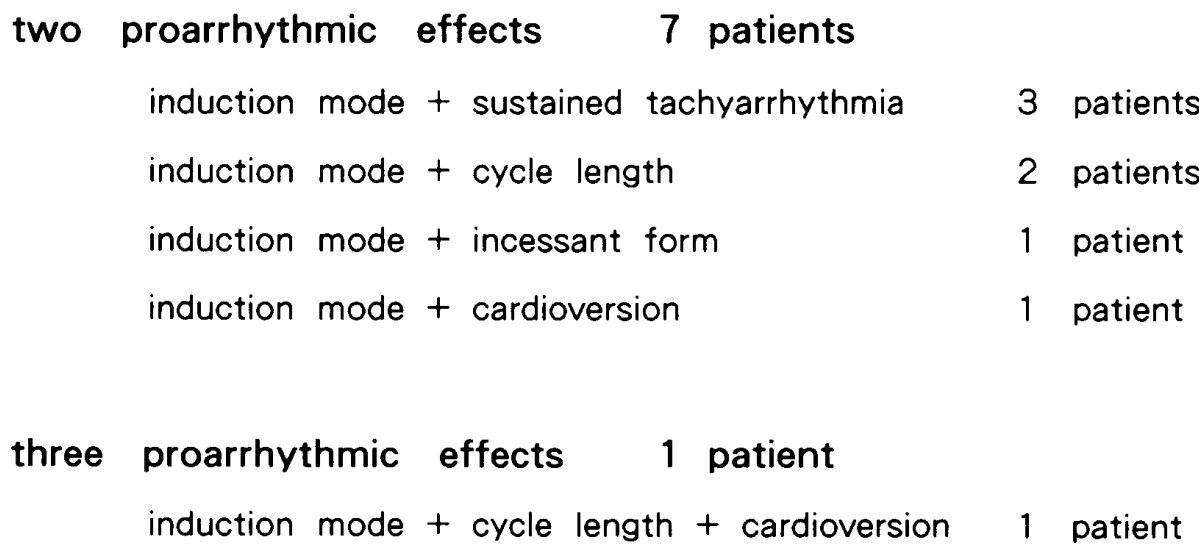

$\begin{array}{lcc}\text { structural heart } & 7 / 8 \text { patients } \\ \text { disease }(+) & (88 \%)\end{array} \quad$ pleomorphic VT $\begin{gathered}6 / 8 \text { patients } \\ (75 \%)\end{gathered}$

Fig.5. Multiple proarrhythmic effects. Seven patients had two proarrhythmic effects and one had three concomitantly. All these 8 patients satisfied criterion 2 (induction mode). Other criteria were shown in this figure. Seven of these 8 patients had structural heart disease and 6 showed pleomorphic VT. For details see text.

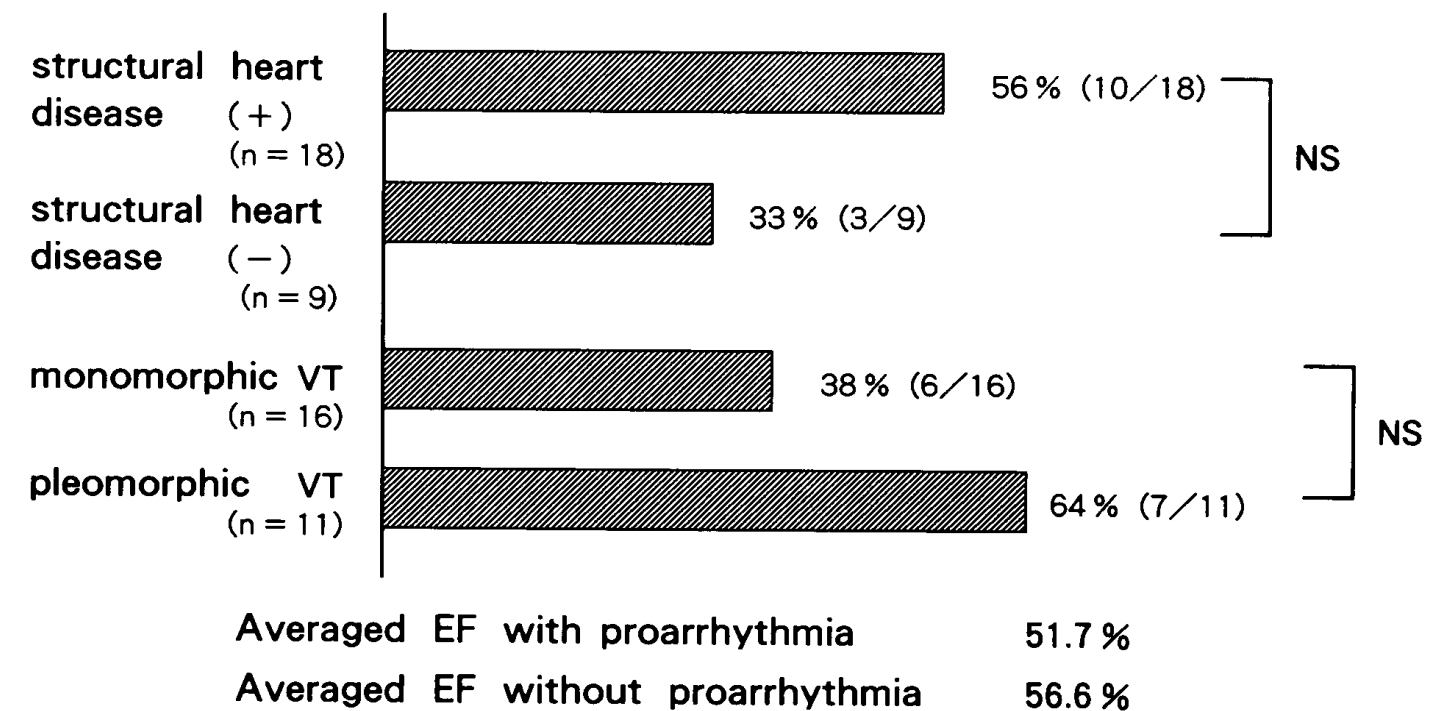

Fig.6. Characteristics of patients. Proarrhythmia developed more easily in patients with structural heart disease. Patients with pleomorphic VTs also had high incidence of proarrhythmia. $n=$ number of cases. $E F=$ ejection fraction of the left ventricle. $\mathrm{NS}=$ not significant.

square test was used and a $p$ value of less than 0.05 was considered significant.

\section{RESULTS}

1) Incidnece of proarrhythmic effects.

Figure 1 shows the incidence of each proarrhythmic effect. The VT cycle length shortened $50 \mathrm{~ms}$ or more with antiarrhythmic drugs in $4(15 \%)$ of the 27 patients and in 6 $(7 \%)$ of the 92 drug testings. A representative case is shown in Fig. 2. In these 6 drug tests, the VT cycle length shortened significnatly from $340 \pm 33 \mathrm{~ms}$ in the control to $277 \pm 33 \mathrm{~ms}$ in the drug tests $(\mathrm{p}<0.001)$.

VT was inducible using less aggressive modes by electrical stimulations in 9 patients $(33 \%)$ and in 18 drug tests $(20 \%)$. These 
studies included conversion of induction modes from DVE to SVE in 7 studies, from RP to DVE or SVE in 2, from TVE to other modes in 4 and no need to use ISP for induction in 5. A representative case is shown in Fig. 3.

VT became incessant on administration of drugs in 4 patients $(15 \%)$ and in 4 drug tests $(4 \%)$.

Conversion of nonsustained VT to sustained VT or VF was observed in 3 patients $(11 \%)$ and in 7 drug tests $(8 \%)$. Because of hemodynamic deterioration, 2 patients $(7 \%)$ required cardioversion to terminate $\mathrm{VT}$ during the drug tests. In other patients, either electrical stimulation or intravenous drug infusion was given to terminate VT.

In total, one or more of the proarrhythmic effects were observed in $13(48 \%)$ of the 27 patients and in $30(33 \%)$ of the $92 \mathrm{EP}$ studies. One proarrhythmic effect was observed in 5 patients. Two proarrhythmic effects concomitantly were observed in 7 patients and three effects were observed in 1 patient. There were 7 EP studies which showed two proarrhythmic effects at the same time. Therefore, total incidence of proarrhythmic effects became 22 patients and 37 EP studies as shown in Fig. 1.

2) Incidence of proarrhythmia vs antiarrhythmic drugs (Fig. 4).

Thirty-six studies were performed with class Ia drugs alone and proarrhythmic effects were recognized in $10(28 \%)$ of these 36 studies. In the same way, proarrhythmic effects were observed in $3(33 \%)$ of the 9 in class Ib, in $1(25 \%)$ of the 4 in class Ic, in 4 $(80 \%)$ of the 5 in class III, and in $1(11 \%)$ of the 9 in class IV drug tests. Proarrhythmic effects were also observed in $5(50 \%)$ of the 10 studies in the combination therapy of class Ia and Ib drugs, in $2(67 \%)$ of the 3 studies in the combination of class Ia and class III drugs, and in all 4 studies in the combination of two of the class Ia drugs. And 12 studies were performed in other combinations, but no proarrhythmic effects were observed in them. These results are shown in Fig. 4.

3) Multiple proarrhythmic effects (Fig. 5).

Two proarrhythmic effects were concomitantly observed in 7 and three effects in 1 of 27 patients. In all of these 8 patients, VT was induced by a less aggressive mode during drug test (criterion 2). Other proarrhythmic effects included the conversion of nonsustained VT to sustained tachyarrhythmia in 3 patients, the shortening of VT cycle length in 3, the development of incessant VT in 1 , and requirement of cardioversion to terminate VT in 2 patients. Seven $(88 \%)$ of these 8 patients had structural heart diseases and $6(75 \%)$ had documentation of pleomorphic VTs. The average ejection fraction (EF) of these 8 patients was $44.1 \%$, which was significantly lower than that of the other 19 patients $(58.5 \% \mathrm{p}<0.05)$.

4) Clinical features of patients with proarrhythmia (Fig. 6).

Proarrhythmic effects occurred in 10 $(56 \%)$ of 18 patients ( $=70 \mathrm{EP}$ studies) who had structural heart diseases, while it occurred in $3(33 \%)$ of 9 patients (=22 EP studies) in those without structural heart diseases. The differences in the numbers of proarrhythmias attributable to antiarrhythmic drugs may be one reason for the high incidence of proarrhythmic events in patients with structural heart diseases who experienced drug refractory VT. Furthermore, the class III drug was only used for drug-resistant VT in patients with structural heart diseases, and the class IV drug was selectively used for idiopathic VT. Therefore, the incidence of proarrhythmia during the use of each drug may be affected by the characteristics of particular forms of VT.

Sixteen patients had single monomorphic VT. Proarrhythmia occurred in $6(38 \%)$ of these patients, while on the other hand, proarrhythmia was observed more frequently in $7(64 \%)$ of the 11 patients who had pleomorphic VTs, but there was no statistical difference between the two groups. The average ejection fraction of the left ventricle in patients with proarrhythmia was slightly lower than that in patients without proarrhythmia, but the statistical difference was not significant between these two groups $(51.7 \%$ vs. $56.6 \%: \mathrm{p}>0.1)$

\section{DISCUSSION}

The standard ECG recording, Holter monitoring, exercise testing, and the EP study are used as methods to evaluate proar- 
rhythmic effects during antiarrhythmic drug therapy of VT!10,13,25 In the standard ECG, the prolongation of the QRS complex or QT interval may be important. Especially, a remarkable prolongation of the QT interval is well known to be associated with the development of Torsades de Pointes. ${ }^{26-28}$ However, a minor change of the QRS duration or QT interval may not be useful to predict the drug efficacy or the development of proarrhythmic effects ${ }^{26-28}$ Since we were careful in our use of antiarrhythmic drugs, no patient showed excessive prolongation of the QT interval (QT interval less than 550 ms) in our study.

Since VT could be induced and terminated by electrical stimulation, and the phenomenon of transient entrainment was frequently confirmed, $29-31$ the mechanism of VT in these patients is supposed to be due to reentry. The development of the proarrhythmia in VT may be closely related to this mechanism. The mechanisms of proarrhythmia can be considered as follows: (1) a widening of the VT induction zone of the single reentry circuit, (2) a shift of the exit site of the single reentry circuit, (3) a modification of anatomical pathways within the reentry circuit, and (4) establishment of another reentrant circuit $32-35$

Criteria of proarrhythmia may not be uniform, but it was arbitrarily classified by us into five categories. Among these, acceleration of the VT rate (shortening of the VT cycle length) has clinical importance since patients may suffer from hemodynamic collapse.$^{2-24}$ In this study, the acceleration of VT was observed in 4 patients $(15 \%)$ and in 6 studies $(7 \%)$. Drugs used in these 6 studies included 3 cases of class Ia, 1 of class Ib, 1 of class III and 1 of class IV regimen. The incidence of acceleration of VT in the present study seems to be the same as in other reports ${ }^{13,23}$ Aggravation of VT into an incessant form or the inability to terminate VT by programmed stimulation may also be of some clinical importance. Especially for these patients, intensive care was given until the serum concentration of drug $(s)$ reached the therapeutic level. In this study, the incessant form was observed in 4 patients $(15 \%)$ and 4 studies (4\%). Special care was taken at bedside for $6-24 \mathrm{~h}$ after treatment. Cardioversion was required for termination of VT in $2(7 \%)$ patients and 2 studies $(2 \%)$. These 6 studies ( 4 of incessant VT and 2 of required cardioversion) included 3 studies using class Ia, and one study using class Ib, class Ic and class III regimen respectively.

It is still debatable whether a mere change of the induction mode of VT to a less aggressive one should be considered as proarrhythmia or not. Previous reports showed that variability exists both in terms of induction of ventricular tachycardia $(11 \%$ to $75 \%)$ and the mode of induction ( $27 \%$ to $73 \%)^{36-39}$ Including these variabilities, we have to consider the benefit or detriment of the drugs' effects. In our study, conversion of the induction mode to a less aggressive one was observed in 9 patients $(33 \%)$ and 8 of these 9 patients had other proarrhythmias at the same time 10,40 Though the significance of the change of the induction mode of VT with regard to long-term prognosis might still be controversial ${ }^{41}$ such change may be closely related to the VT-triggering zone.

Patients with structural heart disease may have extensive arrhythmogenic substrate with the possibility of developing pleomorphic VTs from multiple sites of origin, and antiarrhythmic drugs may establish reentrant circuits in other sites. Furthermore, patients who had 2 or more proarrhythmic responses may have impaired cardiac function due to underlying heart diseases ${ }^{10-13}$

Proarrhythmia has been reported to be related to the administration of many drugs, including new experimental ones26-28 In our study, proarrhythmia was recognized in $13(48 \%)$ of the 27 patients and in $30(33 \%)$ of the 92 studies when it was assessed in the EPS. Such adverse effects could not have been predicted from clinical data and it is only when VT with certain proarrhythmic response occurs spontaneously that they could be recognized. For the early diagnosis of potential hazards of proarrhythmia, EPS will be very useful in the management of recurrent sustained VT.

\section{Possible limitations and implications}

Usually, the occurrence or induction of VT with a different morphology from that of the clinical VT is regarded as proarrhythmia. However, it is debatable whether the phenomenon is truly proarrhythmia. If there are multiple potential reentrant circuits, it is 
possible that a drug can prevent the induction of VT from a specific reentrant circuit, and VT may still arise from other foci on which the drug is not effective. In the latter case, drugs may work to facilitate the establishment of reentrant circuits. Therefore, in the future, it may be necessary to evaluate the drug efficacy in each reentrant circuit.

\section{Acknowledgment}

This work was supported by the Japan Heart Foundation and the IBM Research Fund for 1987 and the 22nd Reserch Grant from the of Mitsui Life Insurance Foundation.

\section{REFERENCES}

1. WELLENS HJJ, BAR FW, LIKE KI, DUREN D, DOHMAN HJ: Effect of procainamide, propranorol, and verapamil on mechanisms of tachycardia in patients with chronic recurrent ventricular tachycardia. Am J Cardiol 40: 579, 1977

2. KIENZLE MG, WILLIAMS PD, ZYGMONT D, DOHELTY JU, JOSEPHSON ME: Antiarrhythmic drug therapy for sustained ventricular tachycardia. Heart and Lung 13: 614, 1984

3. AIZAWA Y, SATOH M, NIWANO S, et al: Clinical, Pharmacological, and Electrophysiological Evaluation of Sustained Ventricular Tachycardia. Proceeding of International Symposium on Cardiac Excerpta Medica p161, 1986 Arrhythmias p137, 1986

4. HARKEN AH, JOSEPHSON ME: Surgical Management of Ventricular Tachycardia. In Tachycardias, ed by JOSEPHSON ME and WELLENS HJJ, Philadelphia, Lea \& Febiger, p.475, 1984

5. ODA H, AIZAWA Y, MURATA $M$, et al: A Successful Electrical Ablation of Recurrent Sustained Ventricular Tachycardia in A Postoperative Case of Tetralogy of Fallot. Jpn Heart $J$ 27: 421, 1986

6. HARTZLER GO: Electrical Catheter Ablation of Refractory Focal Ventricular Tachycardia. J Am Coll Cardiol 2: 1107, 1983

7. CANNOM DS, WINKLE RA: Implantation of the Automatic Implantable Cardioverter Defibrillator(AICD): Practical Aspects. PACE 9: 793, 1986

8. MIROWSKI M: The Automatic Implantable Cardioverter Defibrillator: An Overview. J Am Coll Cardiol 6: 461, 1985

9. KLLY PA, CANNOM DS, GARAN H, et al: The Automatic Implantable Cardioverter Defibrillator: Efficacy, Complications and Survival in Patients With Malignant Ventricular Arrhythmias. $J$ Am Coll Cardiol 11: 1278, 1988

10. RAE AP, KAY HR, HOROWITZ LN, SPIELMAN SR, GREENSPAN AM: Proarrhythmic effects of Antiarrhythmic Drugs in Patients With Malignant Ventricular Arrhythmias Evaluated by Electrophsiologic Testing. J Am Coll Cardiol 12: 131,1988

11. TORRES V, FLOWERS D, SOMBERG JC: The arrhythmogenicity of antiarrhythmic agents. Am Heart J 109: 1090, 1985

12. VELEBIT V, PODRID P, LOWN B, COHEN BH, GRABOYS TB: Aggravation and Provocation of Ventricular Arrhythmias by Antiarrhythmic Drugs. Circulation 65: 8861982

13. POSTER RF, PODRID PJ, LOMBARDI F, LOWN B: Aggravation of arrhythmia induced with antiarrhythmic drugs during electrophsiologic testing. Am Heart J 110: 9, 1985

14. WELLENS HJJ, SCHUILENBURG RM, DURRER D: Electrical stimulation of the Heart in Patients with Ventricular Tachycardia. Circulation 46: 216,1972

15. JOSEPHSON ME, HOROWITZ LN, FARSHIDI A. KASTOR JA: Recurrent sustained ventricular tachycardia. I. Mechanism. Circulation 57: 431, 1978

16. BUXTON AE, WAXMAN HL, MARCHLINSKI FE, UNTEREKER WJ, WASPE LE, JOSEPHSON ME: Role of triple extrastimuli during electrophsiologic study of patients with documented sustained ventricular tachyarrhythmias. Circulation 69: 532,1984

17. VAUGHAN-WILLIAMS EM: A Classification of Antiarrhythmic Actions Reassessed After a Decade of New Drugs. J Clin Pharmacol 24: 129 , 1984

18. VAUGHAN-WILLIAMS EM: Classification of antiarrhythmic drugs. In SANDOE $\mathrm{E}$ FLENSTED-JENSEN E, OLESON HK, eds: Symposium on cardiac arrhythmias. Sodertalje, Sweden, AB Astra, p449, 1970

19. BUXTON AE, MARCHLINSKI FE, DOHERTY $J U$, et al: Repetitive monomorphic ventricular tachycardia: clinical and electrophysiologic characteristics in patients with and without organic heart disease. Am J Cardiol 54: 997, 1984

20. LIN FC, FINLEY CD, RAHIMTOOLA SH, et al: Idiopathic paroxysmal ventricular tachycardia with a QRS pattern of right bundle branch block and left axis deviation; a unique clinical entity with specific properties. Am J Cardiol 52: 95, 1983

21. OHE T, SHIMOMURA K, AIHARA N, et al: Idiopathic sustained left ventricular tachycardia; clinical and electrophysiologic charcteristics. Circulation 77: 560, 1988

22. HOROWITZ LN, ZIPES DP, BIGGER JT Jr, CAMPBELL RWF, MORGANROTH J, PODRID PJ, ROSEN MR, WOOSLEY RL: Proarrhythmia, Arrhythmogenesis or Aggravation of Arrhythmia A Status Report, 1987. Am J Cardiol 59: 54E, 1987

23. CAMPBELL RWF: Arrhythmogenesis A European Perspective. Am J Cardiol 59: 49E, 1987

24. ZIPES DP: Proarrhythmic Effect of Antiarrhythmic Drugs. Am J Cardiol 59: 26E, 1987

25. PODRID PJ, LAMPERT S, GRABOYS TB, BLATT CM, LOWN B: Aggravation of Arrhythmia by Antiarrhythmic Drugs-Incidence and Predictors. Am J Cardiol 59: 38E, 1987

26. STRASBERG B, SCLAROVSKY S, ERDBERG A, DUFFY CE, LAM W, SWIRYN S, AGMON J, ROSEN KM: Procainamide-Induced Polymorphous ventricular Tachycardia. Am J Cardiol 47: 1309,1981 
27. COCCO G, STROZZI C, CHU D, PANSINI R: Torasades de pointes as a manifestation of mexiletine toxicity. Am Heart J 100: 878, 1980

28. SCLAROVSKY S, LEWIN RF, KRACOFF O, STRASBERG B, ARDITTI A, AGMON J: Amiodarone-induced polymorphous ventricular tachycardia. Am Heart J 105: 6, 1983

29. WALDO AL, PLUMB VJ, ARCINIEGAS JG, MACLEAN WAH, COOPER TB, PRIEST MF, JAMES TN: Transient Entrainment and Interruption of the Atrioventricular Bypass Pathway Type of Paroxysmal Artial Tachycardia. Circulation 67: 73,1983

30. AIZAWA Y, ODA H, SATOH M, MURATA M, SHIBATA A, EGUCHI S: Transient entrainment of ventricular tachycardia with continuous local electrical activity. Am Heart J 114: 182, 1987

31. AIZAWA Y, MURATA M, SATHO M, FUNAZAKI T, MATUOKA A, SHIBATA A, EGUCHI S: 53rd Annual Meeting of Japan Circulation Society Symposium III Requirements of Non-pharmacologic Interventions in the Treatment of Recurrent Sustained Ventricular Tachycardia. Jpn Circ J 53: 1989 (in press)

32. STANTON MS, PRYSTOWSKY EN, FINEBERG NS, MILES WN, ZIPES DP, HEGER JJ: Arrhythmogenic Effect of Antiarrhythmic Drugs: A Study of 506 Patients Treated for Ventricular Tachycardia or Fibrillation. J Am Coll Cardiol 14: 209, 1989

33. MORGANROTH J: Risk Factors for the Developement of Proarrhythmic Events. Am J Cardiol 59: 32E, 1987

34. GOLDSTEIN RE, TIBBITS PA, OETGEN WJ: Proarrhythmic Effets of Antiarrhythmic Drugs. Ann NY Acad Sci 427: 94-100, 1984
35. WOOSLEY RL, RODEN DM: Pharmacologic Cause of Arrhythmogenic Actions of Antiarrhythmic Drugs. Am J Cardiol 59: 19E, 1987

36. COOPER MJ, HUNT LJ, PALMER KJ, DENNISS AR, RICHARDS DA, UTHER JB, ROSS DL: Quantitation of Day to Day Variability in Mode of Induction of Ventricular Tachyarrhythmia by Programmed Stimulation. J Am Coll Cardiol 11: 101,1988

37. COOPER MJ, HUNT LJ, RICHARDS DA, DENNISS AR, UTHER JB, ROSS DL: Effect of Repetition of Extrastimuli on Sensitivity and Reproducibility of Mode of Induction of Ventricular Tachycardia by Programmed Stimulation. $J \mathrm{Am}$ Coll Cardiol 11: 1260, 1988

38. BECKMAN KJ, VELASCO CE, KRAFCHEK J, LIN HT, MAGRO SA, WYNDHAM CRC: Significant variability in the mode of ventricular tachycardia induction and its implications for interpretation of acute drug testing. Am Heart $J$ 116: 718, 1988

39. MCPHERSON CA, ROSENFELD LE, BATSFORD WP: Day-to-Day Reproducibility of Responses to Right Ventricular Programmed Electrical Stimulation: Implications for Serial Drug Testing. Am J Cardiol 55: 689, 1985

40. HOROWITZ LN, GREENSPAN AM, RAE AP, KAY HR, SPIELMAN SC: Proarrhythmic Responses During Electrophysiologic Testing. Am J Cardiol 59: 45E, 1987

41. BORGGREFE M, TPAMPISCH HJ, BREITHARDT G: Reappraisal of Criteria for Assessing Drug Efficacy in Patients With Ventricular Tachyarrhythmia; Complete Versus Partial Suppression of Inducible Arrhythmia. J Am Coll Cardiol 12: 140, 1988 